SUBJECT AREAS:

ELECTRONIC DEVICES

APPLIED PHYSICS

Received

24 December 2013

Accepted

6 March 2014

Published

21 March 2014

Correspondence and requests for materials should be addressed to Z.Y.L. (zyli@ustc.edu. cn) or W.C. (phycw@ nus.edu.sg)

* These authors contributed equally to this work.

\section{Elementary Process for CVD Graphene on Cu(1 10): Size-selective Carbon Clusters}

Jialin Zhang ${ }^{1 *}$, Zhunzhun Wang ${ }^{2 *}$, Tianchao Niu ${ }^{3}$, Shengnan Wang ${ }^{2}$, Zhenyu $\mathrm{Li}^{2} \&$ Wei Chen ${ }^{1,3}$

\begin{abstract}
'Department of Physics, National University of Singapore, 2 Science Drive 3, 1 17542, Singapore, ${ }^{2}$ Hefei National Laboratory for Physical Sciences at Microscale, University of Science and Technology of China, Hefei 230026, China, ${ }^{3}$ Department of Chemistry, National University of Singapore, 3 Science Drive 3, 117543, Singapore.
\end{abstract}

Revealing the graphene growth mechanism at the atomic-scale is of great importance for achieving high quality graphene. However, the lack of direct experimental observation and density functional theory (DFT) verification hinders a comprehensive understanding of the structure of the carbon clusters and evolution of the graphene growth on surface. Here, we report an in-situ low-temperature scanning tunneling microscopy (LT-STM) study of the elementary process of chemical vapor deposition (CVD) graphene growth via thermal decomposition of methane on $\mathrm{Cu}(110)$, including the formation of monodispersed carbon clusters at the initial stage, the graphene nucleation and the ripening of graphene islands to form continuous graphene film. STM measurement, supported by DFT calculations, suggests that the carbon clusters on the surface are $\mathrm{C}_{2} \mathrm{H}_{5}$. It is found that graphene layers can be joined by different domains, with a relative misorientation of $30^{\circ}$. These graphene layers can be decoupled from $\mathrm{Cu}(110)$ through low temperature thermal cycling.

西 ver since its mechanical exfoliation from small mesas of highly oriented pyrolytic graphite, graphene has spurred a tremendous of interest because of its exceptional electronic and mechanical properties, such as anomalous quantum Hall Effect (QHE), long-range ballistic transport, high carrier mobility, tunable band gap, high elasticity and intrinsic strength $\mathrm{h}^{1-4}$. All these qualify graphene as a promising material for applications in microelectronic and spintronic devices ${ }^{1-3}$, sensors ${ }^{5}$, supercapacitors ${ }^{6}$, building blocks for multifunctional composites $^{7}$ as well as for structural and mechanical applications $s^{4}$. Motivated by these extraordinary properties and numerous potential applications, a number of graphene fabrication methods have been explored, including the micromechanical cleavage of graphite ${ }^{1}$, thermal decomposition of $\mathrm{SiC}^{8}$, reduction of chemically functionalized graphene ${ }^{9-12}$, chemical exfoliation of graphite ${ }^{13}$ and transition metal (TM)-catalyzed chemical vapor deposition $(\mathrm{CVD})^{14-26}$, and so on. Graphene prepared by cleavage and exfoliation of graphite shows superior transport properties, but its size is usually limited to micrometers and the productivity of this method is very low ${ }^{1}$. Epitaxial graphene on $\mathrm{SiC}$ allows larger area synthesis ${ }^{27}$, but this method induces noticeable densities of defects and achieving large graphene domains with uniform thickness remains a challenge ${ }^{28}$. Transition metal assisted growth of graphene, which provides many unique advantages, such as industrial scalability ${ }^{21}$, relatively low temperature processing ${ }^{18}$, easy transferring onto other substrates ${ }^{23}$, has received the most attention.

The graphene growth on TM surfaces is based on high-temperature pyrolysis of hydrocarbons and different growth mechanisms can be involved according to the carbon solubility limit in the metal. For the growth on TM where carbon is soluble, the graphene forms when the sample is cooled and carbon segregates on the surface (surface segregation ) $^{29-32}$; but for TM with very low carbon solubility, the synthesis is limited to the surface of the catalyst and mainly involves surface diffusion and nucleation of carbon atoms. The growth of graphene can be accomplished by CVD via two approaches: directly cracking the carbon source on TM surface at a high temperature or temperature programmed growth (TPG) via room temperature adsorption of the molecules followed by pyrolysis and graphene growth at a fixed elevated temperature ${ }^{17}$.

Graphene growth has been demonstrated on a variety of TMs. For example, Li and colleagues reported a CVD method that used copper-foil to produce single crystal graphene with dimensions of up to $0.5 \mathrm{~mm}^{20}$; Bae and col leagues demonstrated a roll-to roll production of 30 inch graphene films for transport electrodes ${ }^{21}$; Gao and colleagues showed the repeated growth and bubbling transfer of graphene with millimeter-size single-crystal grains using platinum ${ }^{24}$. Additionally, intensive theoretical efforts have been devoted to revealing the growth 
mechanism ${ }^{14,33-43}$. Using first-principle calculations, Chen et al. found on flat surfaces of $\operatorname{Ir}(111)$ and $\operatorname{Ru}(0001)$, two carbon atoms repel each other; while they prefer to form a dimer on $\mathrm{Cu}(111)^{37}$. Zhang et al. also revealed that $\mathrm{C}_{2} \mathrm{H}_{2}$ can be easily formed on a $\mathrm{Cu}(111)$ surface, which represents a more favorable reaction path compared to $\mathrm{CH}$ dissociation ${ }^{38}$. By careful optimization of the supported carbon clusters $\mathrm{C}_{\mathrm{N}}$ on $\mathrm{Ni}(111)$, Gao et al. indicated a ground state structure transition from a one-dimensional (1D) carbon chain to a two-dimensional (2D) $\mathrm{sp}^{2}$ carbon network at $\mathrm{N} \sim 10-12^{40}$; while Wesep and co-workers proposed an energetic preference for the formation of stable 1D carbon nanoarches consisting of 3-13 atoms on $\mathrm{Cu}(111)$ surface ${ }^{43}$. Explored by ab initio calculations, Yuan et al. showed that the core-shell $\mathrm{C}_{21}$ is a very stable magic carbon cluster on $\mathrm{Rh}(111), \mathrm{Ru}(0001), \mathrm{Ni}(111)$ and $\mathrm{Cu}(111)$ surfaces $^{42}$. Zangwill et al. predicted that an immobile island composed of six five-atom carbon clusters as the smallest stable precursor to graphene growth on metals ${ }^{41}$. Despite these inspiring achievements, most of these theoretical studies only address the number of carbon atoms, and the precise determination of hydrogen atoms within the cluster is rare. Moreover, very little of the growth mechanism in the initial nucleation stages of carbon atoms has been revealed experimentally ${ }^{15,16,44}$. In this regard, atomic-scale characterization of a complete process of graphene growth in combination with theoretical calculations is of great importance, for both fundamental interest and achieving high quality graphene.

Here, we report an atomic scale characterization of the elementary process of CVD graphene growth via thermal decomposition of methane $\left(\mathrm{CH}_{4}\right)$ on $\mathrm{Cu}(110)$ using low-temperature scanning tunneling microscopy (LT-STM), including the formation of monodispersed carbon clusters at low temperature, nucleation and ripening of graphene islands at high temperature. Combined with first principles calculations, the monodispersed carbon clusters are identified as $\mathrm{C}_{2} \mathrm{H}_{5}$. Different domains stitch together to form a graphene layer, with a preference angle of $30^{\circ}$ at the grain boundaries. These graphene layers can be decoupled from $\mathrm{Cu}(110)$ through low temperature thermal cycling.

\section{Results}

As shown by the high magnification STM image in Fig. 1a, upon the deposition of $\mathrm{CH}_{4}$ at room temperature (RT) and subsequent annealing at $480^{\circ} \mathrm{C}$ in $\mathrm{CH}_{4}$ at a pressure of $2 \times 10^{-5} \mathrm{mbar}$ for $50 \mathrm{~min}$, the $\mathrm{Cu}(110)$ surface was almost decorated with carbon clusters of monodispersed size. Each carbon cluster appears as a bright spot with an identical size of $0.4 \mathrm{~nm}$. Careful inspection of the STM image reveals that the surface is decorated by isolated but well-defined superstructures, where the carbon clusters are adsorbed in an epitaxial relationship with the underling $\mathrm{Cu}(110)$. As indicated by the dashed lines in Fig. $1 \mathrm{a}$, the minimum distance between two neighboring row is $2 \mathrm{a}_{0}$ of $0.512 \mathrm{~nm}$; while it is $2 \mathrm{~b}_{0}$ of $0.723 \mathrm{~nm}$ between two columns ( $\mathrm{a}_{0}$ and $\mathrm{b}_{0}$ are the unit cell dimensions of $\left.\mathrm{Cu}(110)\right)$. It can also be revealed that the carbon cluster arrays are aligned precisely with the crystal orientation of the underlying $\mathrm{Cu}(110)$. The carbon cluster at this low coverage was referred to as "cluster 1 " with a density around $2.70 \times$ $10^{14} / \mathrm{cm}^{2}$. Previous theoretical studies proposed that carbon dimmers are energetically favorable on the $\mathrm{Cu}$ surface $\mathrm{e}^{37-39}$. Therefore, we tentatively assign these carbon clusters as carbon dimers $\left(\mathrm{C}_{2} \mathrm{H}_{\mathrm{x}}\right)$.

Further increasing the coverage of the carbon clusters can result in the formation of a hexagonally close packed structure, as shown in Fig. 1b. The coverage of the carbon clusters can be increased through low temperature thermal cycling as described in the supporting information. Some gaps can still be observed between the ordered domains. However, the carbon clusters in each ordered domain posses the unit cell with $\mathrm{a}=0.515 \mathrm{~nm}, \mathrm{~b}=0.500 \mathrm{~nm}$ and an inclusion angle of $60^{\circ}$, as indicated by arrows A and B. Upon saturation of the carbon clusters on the surface, they formed highly ordered close packed structure over the surface, as shown in Fig. 1c. The unit cell
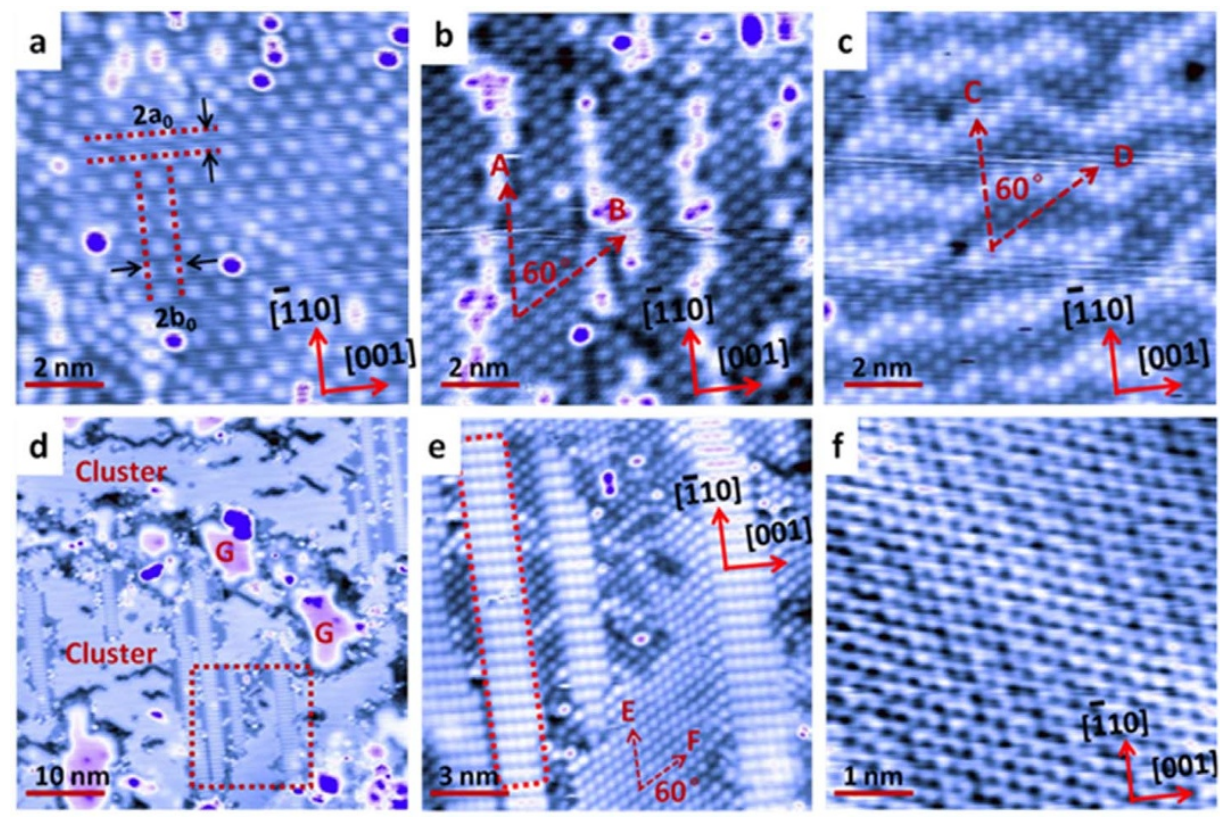

Figure 1 Evolution of carbon clusters and formation of grapheme on $\mathrm{Cu}(110)$. (a) STM image $\left(\mathrm{V}_{\text {tip }}=0.25 \mathrm{~V}, 10 \times 10 \mathrm{~nm}^{2}\right)$ of the low coverage carbon clusters on $\mathrm{Cu}(110)$, which were formed upon the deposition of $\mathrm{CH}_{4}$ at room temperature and subsequent annealing at $480^{\circ} \mathrm{C}$ in $\mathrm{CH}_{4}$ at a pressure of $2 \times 10^{-5}$ mbar for $50 \mathrm{~min}$. (b) STM image $\left(\mathrm{V}_{\text {tip }}=1 \mathrm{~V}, 10 \times 10 \mathrm{~nm}^{2}\right)$ of carbon clusters at higher coverage; A and B indicate the direction of the unit cell vectors. (c) Hexagonally close packed cluster structure formed by further increasing the carbon clusters coverage. $\left(\mathrm{V}_{\text {tip }}=0.2 \mathrm{~V}, 10 \times 10 \mathrm{~nm}{ }^{2}\right), \mathrm{C}$ and $\mathrm{D}$ indicate the direction of the unit cell vectors. (d) Large scale STM image $\left(V_{\text {tip }}=0.5 \mathrm{~V}, 50 \times 50 \mathrm{~nm}^{2}\right)$ of carbon clusters and small graphene flakes on $\mathrm{Cu}(110)$ by annealing $\mathrm{Cu}(110)$ in $\mathrm{CH}_{4}$ at $550^{\circ} \mathrm{C}$ at a pressure of $2 \times 10^{-5} \mathrm{mbar}$ for $130 \mathrm{~min}$, where the graphene flakes are indicated by " $\mathrm{G}$ ".

(e) The corresponding high resolution STM image $\left(\mathrm{V}_{\text {tip }}=0.1 \mathrm{~V}, 15 \times 15 \mathrm{~nm}^{2}\right)$ showing the hexagonally close packed carbon clusters in panel 1 (d), where $\mathrm{E}$ and $\mathrm{F}$ indicate the direction of the unit cell vectors. (f) The atomically resolved STM image $\left(\mathrm{V}_{\text {tip }}=0.03 \mathrm{~V}, 5 \times 5 \mathrm{~nm}^{2}\right)$ showing the $1 \times 1$ graphene lattice. 
was further reduced to $c=0.450 \mathrm{~nm}, \mathrm{~d}=0.480 \mathrm{~nm}$ with an unchanged inclusion angle of $60^{\circ}$. At this stage, the carbon cluster density was increased to $10.9 \times 10^{14} / \mathrm{cm}^{2}$, referred to as "cluster 2 ". In this regime, the arrangement is supposed to be cluster-cluster interaction dominated. Some brighter lines can be frequently observed, induced by the stress relaxation at high cluster coverage with increased lateral inter-cluster interaction.

Annealing the $\mathrm{Cu}(110)$ surface at high temperature at $550^{\circ} \mathrm{C}$ in $\mathrm{CH}_{4}$ at a pressure of $2 \times 10^{-5} \mathrm{mbar}$ for $130 \mathrm{~min}$ can promote the nucleation of small graphene flakes. As shown in Fig. 1d, at this stage the carbon clusters co-exist with the small graphene flakes which are indicated as "G". The high magnification STM image in Fig. 1e reveals that the clusters on $\mathrm{Cu}(110)$ are "cluster 2 ". The directions of the unit cell are indicated by arrows $\mathrm{E}$ and $\mathrm{F}$, with lateral dimensions of $\mathrm{e}=0.450 \mathrm{~nm}, \mathrm{f}=0.480 \mathrm{~nm}$ and an inclusion angle of $60^{\circ}$. The bright stripes inserted between these clusters are clean $\mathrm{Cu}(110)$ surface but with a $1 \times 2$ superstructure as highlighted by the red dotted line in Fig. 1e. Figure if shows the atomically resolved STM image of the $1 \times 1$ graphene lattice, and the crystal orientation of the underlying $\mathrm{Cu}(110)$ is indicated in the lower right corner.

To obtain the atomic structure of the carbon clusters, the adsorption of various carbon clusters on $\mathrm{Cu}(110)$ were simulated using DFT. First, the stability of $\mathrm{C}_{1} \mathrm{H}_{\mathrm{x}}(0 \sim 4)$ and $\mathrm{C}_{2} \mathrm{H}_{\mathrm{x}}(0 \sim 6)$ clusters on $\mathrm{Cu}(110)$ were studied. We define the formation energy in equation (1)

$$
\mathbf{E}_{\mathrm{F}}=\mathbf{E}_{\mathrm{tot}}-\mathbf{E}_{\mathrm{sub}}-\sum_{\mathbf{i}} \mathbf{n}_{\mathrm{i}} \boldsymbol{\mu}_{\mathrm{i}}
$$

where $E_{\text {tot }}$ is the total energy of the adsorbed system, $E_{\text {sub }}$ is the energy of clean $\mathrm{Cu}(110)$ substrate, $\mu_{\mathrm{i}}$ and $\mathrm{n}_{\mathrm{i}}(\mathrm{i}=\mathrm{C}, \mathrm{H})$ represent chemical potential and the number of atoms in the cluster, respectively. Considering the equilibrium of $\mathrm{CH}_{4}$ and $\mathrm{H}_{2}$, the relationship of $\mu_{\mathrm{H}}$ and $\mu_{\mathrm{C}}$ in unit of electron volt can be obtained as equation (2) by the process described in the supporting information:

$$
\mu_{\mathrm{c}}=\mathrm{g}_{\mathrm{CH} 4}-4 \mu_{\mathrm{H}}=-2 \mu_{\mathrm{H}}-9.695+0.0689 \ln \chi
$$

Here, $\chi$ is the ratio of the partial pressures of $\mathrm{CH}_{4}$ and $\mathrm{H}_{2}$.

For each carbon cluster species, the most stable adsorption configuration was found by checking different adsorption sites on $\mathrm{Cu}(110)$ surface, including the hollow site ( $\mathrm{H}$-site), bridge-long site ( $\mathrm{B}_{\text {long }}$ site), bridge-short site $\left(\mathrm{B}_{\text {short }} \text { site) and Top site (T-site }\right)^{45}$. Figure 2 shows the formation energy of various carbon cluster species

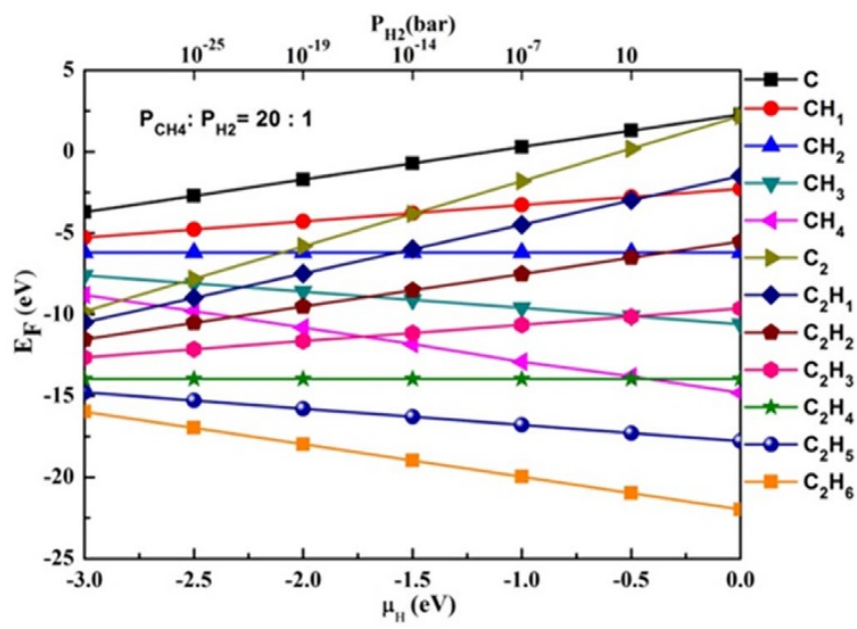

Figure $2 \mid$ Relationship of formation energy and chemical potential of $\mathrm{H}$ or the pressure of $\mathrm{H}_{2}$. Relationship of adsorption energy and chemical potential of $\mathrm{H}$ or the pressure of $\mathrm{H}_{2}$ during CVD growth of carbon-clusters on $\mathrm{Cu}(110)$ at $\mathrm{T}=527^{\circ} \mathrm{C}$. The ratio of partial pressures of $\mathrm{CH}_{4}$ and $\mathrm{H}_{2}$ is $\chi$ $=20$. as a function of the chemical potential of $\mathrm{H}$ (thus the partial pressure of $\mathrm{H}_{2}$ ). The $\chi$ here was set to be $20: 1$; we also tested $\chi=1: 20$, which gave similar results.

From Fig. 2, it is easy to find that clusters $\mathrm{C}_{2} \mathrm{H}_{6}$ and $\mathrm{C}_{2} \mathrm{H}_{5}$ are the two most stable species under all physical $\mathrm{H}_{2}$ partial pressure. Although the formation energy of $\mathrm{C}_{2} \mathrm{H}_{6}$ is very large, as a close shell molecule, its adsorption energy is expected to be very small, and it's hence easy to desorb from $\mathrm{Cu}(110)$ at high temperature. The average lifetime of $\mathrm{C}_{2} \mathrm{H}_{6}$ and $\mathrm{C}_{2} \mathrm{H}_{5}$ can be estimated by their adsorption energy $\mathrm{E}_{\mathrm{a}}$ via $\tau_{a}=\frac{1}{v_{0}} e^{E a / k T}{ }^{46}$. According to our calculations, adsorption energy of $\mathrm{C}_{2} \mathrm{H}_{6}$ and $\mathrm{C}_{2} \mathrm{H}_{5}$ on $\mathrm{Cu}(110)$ surface are 0.41 and $2.85 \mathrm{eV}$, respectively. $v_{0}$ is about $10^{13} \mathrm{~s}^{-1}$. Therefore, their average lifetime on the surface at $480^{\circ} \mathrm{C}$ is $5.5 \times 10^{-11}$ and $1.2 \times 10^{6} \mathrm{~s}$, respectively. Such a short lifetime makes $\mathrm{C}_{2} \mathrm{H}_{6}$ not be able to be observed by STM. Therefore, $\mathrm{C}_{2} \mathrm{H}_{5}$ could be the most possible abundant species from the thermodynamic point of view.

STM images of several partially dehydrogenated carbon dimer species were also simulated using the Tersoff and Hamann approximation $^{47}$. Figure 3 shows the optimized structures and simulated STM images of $\mathrm{C}_{2}, \mathrm{C}_{2} \mathrm{H}_{4}, \mathrm{C}_{2} \mathrm{H}_{5}$ and $\mathrm{C}_{2} \mathrm{H}_{6}$. The optimized unit cell of the carbon cluster is $2 \mathrm{a}_{0}=0.504 \mathrm{~nm}, 2 \mathrm{~b}_{0}=0.713 \mathrm{~nm}$. Among these carbon clusters, the simulated STM image of $\mathrm{C}_{2} \mathrm{H}_{5}$ is in good agreement with the experimental results. All other stable species cannot reproduce the experimental circular shape. Hence, the basic structures of the carbon clusters are elucidated by the STM images in combination with DFT calculations as $\mathrm{C}_{2} \mathrm{H}_{5}$.

Large graphene flakes can be achieved through low temperature thermal cycling process as described in the supporting information. Figure 4a shows a large scale STM image of a flake of graphene film on $\mathrm{Cu}(110)$ interconnected by two graphene grains, forming a grain boundary in between as indicated by the red ellipse. Close-up (Fig. 4b) and the corresponding atomic-resolution STM images (Fig. 4c) reveal that the two graphene grains are stitched together to form a continuous film with a relative misorientation of $30^{\circ}$. The detailed atomic structure at the grain boundary cannot be identified

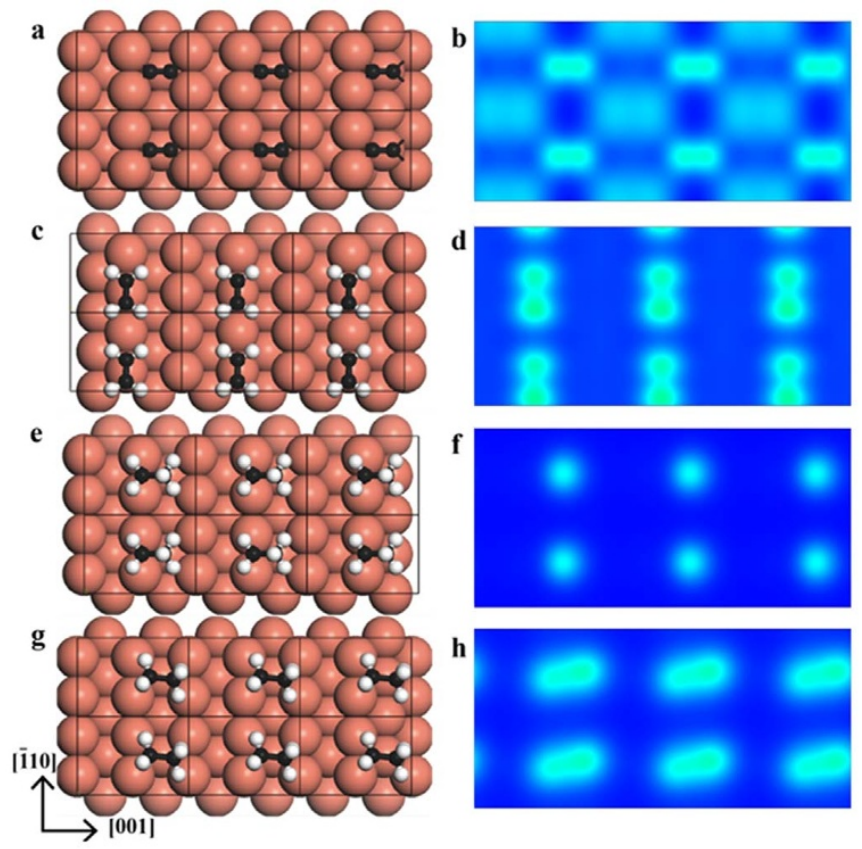

Figure 3 Optimized structures (left panels) and simulated STM images. Optimized structures (left panels) and simulated STM images (right panels) of (a, b) $\mathrm{C}_{2},(\mathrm{c}, \mathrm{d}) \mathrm{C}_{2} \mathrm{H}_{4}$, (e, f) $\mathrm{C}_{2} \mathrm{H}_{5}$, and (g, h) $\mathrm{C}_{2} \mathrm{H}_{6}$. The integrated density of states from $0.25 \mathrm{~V}$ below $\mathrm{E}_{\mathrm{F}}$ to the Fermi level is used to simulate the STM image, which represents the HOMO of the carbon clusters. 

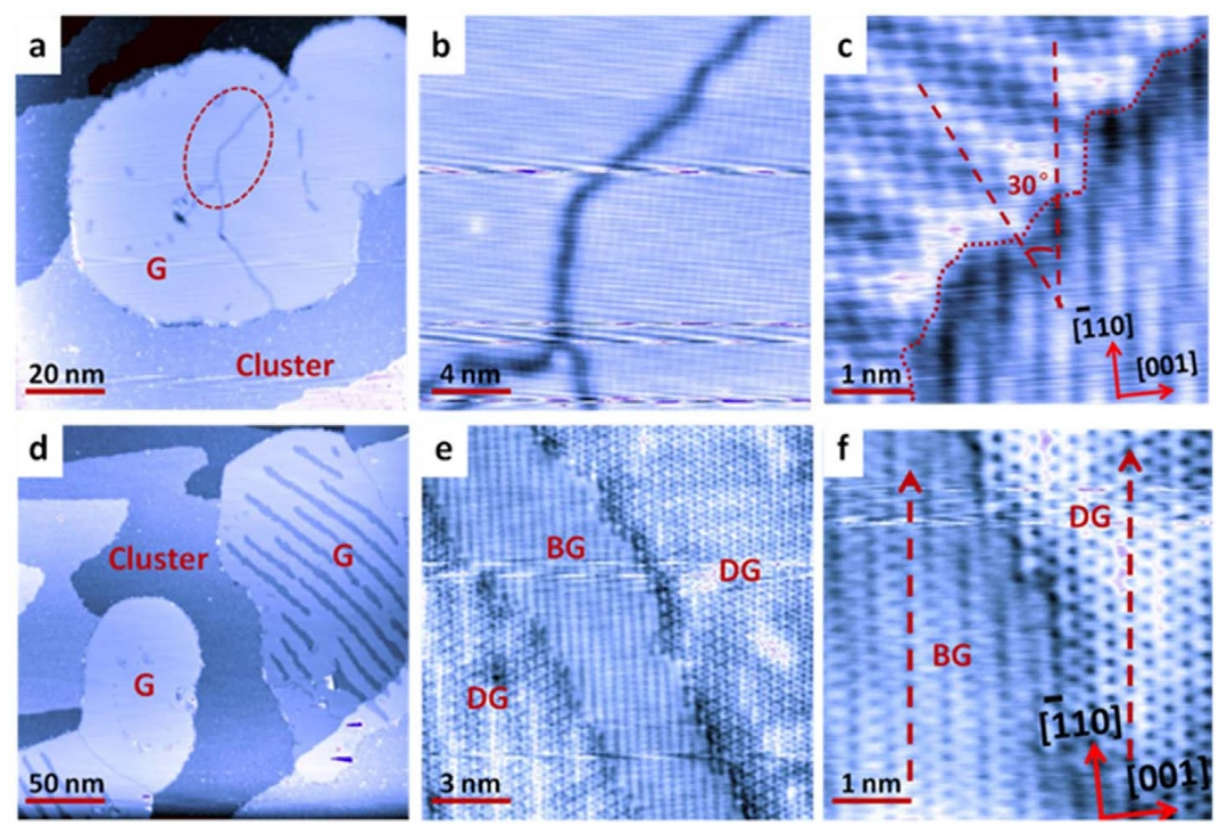

Figure 4 STM images showing the jointed domains and low temperature thermal cycling induced decoupling of graphene from $\mathrm{Cu}(110)$ substrate. (a) Large scale STM image $\left(V_{\text {tip }}=1 \mathrm{~V}, 100 \times 100 \mathrm{~nm}^{2}\right)$ showing one graphene flake jointed by different domains, which was formed by low temperature thermal cycling and subsequent annealing of the carbon clusters on $\mathrm{Cu}(110)$ up to $720^{\circ} \mathrm{C}$. (b) The corresponding high resolution STM image $\left(\mathrm{V}_{\text {tip }}=0.04 \mathrm{~V}, 20 \times 20 \mathrm{~nm}^{2}\right)$ showing the domain boundary. (c) The atomically resolved STM image $\left(\mathrm{V}_{\text {tip }}=0.03 \mathrm{~V}, 5 \times 5 \mathrm{~nm}^{2}\right)$ illustrating two distinct graphene orientations, the upper domain is orientated at an angle of $30^{\circ}$ relative to the lower domain. (d) Large scale $\mathrm{STM}$ image $\left(\mathrm{V}_{\text {tip }}=1.0 \mathrm{~V}\right.$, $250 \times 250 \mathrm{~nm}^{2}$ ) of $\mathrm{Cu}(110)$ covered by large flakes of graphene and carbon clusters. (e) A continuous single layer of graphene with different contrast $\left(\mathrm{V}_{\text {tip }}=0.05 \mathrm{~V}, 15 \times 15 \mathrm{~nm}^{2}\right)$. (f) $\left(\mathrm{V}_{\text {tip }}=-0.01 \mathrm{~V}, 5 \times 5 \mathrm{~nm}^{2}\right)$ The corresponding high resolution STM images of panel (e), where the orientation of graphene is indicated by the red arrows.

from our STM image, but it has been theoretically proposed and experimentally conformed as a series of pentagons, heptagons and distorted hexagons $\mathrm{s}^{25,48}$. The graphene grows in different orientations with respect to the underlying lattice, resulting in two different moiré patterns. As shown in Fig. 4c, the lower right panel shows a moiré superstructure almost aligned with the underlying $\mathrm{Cu}(110)$ lattice, referred to as R0 phase. The graphene lattice of the upper left panel shows a different moiré pattern with a larger periodic modulation and is rotated by $30^{\circ}$ from the lower R0 phase, referred to as R30 phase. Supplementary Fig. S3 on line shows a graphene film joined by multi-domains taken from a different location on $\mathrm{Cu}(110)$, which also shows a $30^{\circ}$ misorientation. The preference of around $30^{\circ}$ misorientation between two domains has also been reported by other groups $^{19,25}$. For graphene grown on $\mathrm{Ru}(0001)$, only one orientation can be observed, due to the strong interaction between graphene and $\mathrm{Ru}^{29}$. The two dominating orientations observed here and the fact that graphene can grow continuously across $\mathrm{Cu}$ step edges could indicate a weaker graphene- $\mathrm{Cu}$ interaction when compared with $\mathrm{Ru}$.

As described in the supporting information, during the experiment, we introduced the low temperature thermal cycling method to increase the carbon cluster coverage. Figure $4 \mathrm{~d}$ shows the STM image of large flakes of graphene coexisting with carbon clusters on $\mathrm{Cu}(110)$. After repeating several cycles of low temperature thermal cycling, the graphene flakes on the surface possess two stripe-shaped contrasts. Comparison between Supplementary Fig. S2 on line and Fig. $4 \mathrm{~d}$ reveals that the appearance of those bright stripes are same with the previous small graphene islands; while the dark stripes are newly produced during the low temperature thermal cycling. Close up STM image in Supplementary Fig. S4 on line and Fig. $4 \mathrm{f}$ reveals that the bright and dark stripes alternated between each other with a continuous boundary. As shown in Fig. 4e, the bright stripes (BG) show moiré pattern resembling the underlying $\mathrm{Cu}(110)$; while the dark stripes (DG) display prefect hexagonal graphene lattice. These contrasts result from the modulation by different interactions with the underlying $\mathrm{Cu}(110)$. The appearance of the prefect hexagonal graphene lattice in DG suggests that the graphene in this region is physically decoupled from the underlying $\mathrm{Cu}(110)$.

The formation of such physically decoupled graphene can arise from the intercalation at the graphene $/ \mathrm{Cu}(110)$ interface by hydrogen atoms released from $\mathrm{CH}_{4}$ decomposition, similar to the previously reported hydrogen ${ }^{49}$, lithium ${ }^{50}$, oxygen ${ }^{51}$, and fluorine intercalation to form quasi-free-standing graphene ${ }^{52}$; or from the strain relief during the annealing/cooling cycles due to the different thermal expansion of graphene film and $\mathrm{Cu}$ substrate ${ }^{53}$. More controlled experiment and detailed theoretical calculations will be carried out to unravel the decoupling mechanism.

\section{Discussion}

Through the combination of the LT-STM and DFT calculations, we reveal the elementary process of graphene growth on $\mathrm{Cu}(110)$ surface via thermal decomposition of $\mathrm{CH}_{4}$. Low temperature annealing $\left(>480^{\circ} \mathrm{C}\right)$ in $\mathrm{CH}_{4}$ results in the formation of carbon clusters at the initial stage; further high temperature annealing $\left(>550^{\circ} \mathrm{C}\right)$ activates the graphene nucleation; prolonged annealing in the absence of $\mathrm{CH}_{4}$ propels the diffusing and ripening of these graphene island to form continuous graphene films extended over the surface. Low temperature thermal cycling induced decoupling of graphene from $\mathrm{Cu}(110)$ has also been demonstrated. Our systematic investigations identify the fundamental carbidic building blocks by STM measurement, and further elucidate their atomic structures through DFT calculations. Our work could lay the foundation for providing rational design rules for synthesis of large area single crystalline graphene films.

\section{Methods}

Growth of graphene on $\mathrm{Cu}(\mathbf{1 1 0})$. Graphene was grown on a single crystal $\mathrm{Cu}(110)$ via thermal decomposition of $\mathrm{CH}_{4}$. Prior to the deposition of $\mathrm{CH}_{4}, \mathrm{Cu}(110)$ substrate was cleaned by a few cycles of $\mathrm{Ar}^{+}$ion bombardment and subsequent annealing at $530^{\circ} \mathrm{C}$. The $\mathrm{CH}_{4}$ gas was introduced into the growth chamber through a leak valve, and the pressure was monitored by a cold cathode gauge. A typical growth procedure 
is as follows: the $\mathrm{Cu}(110)$ substrate was exposed to $\mathrm{CH}_{4}$ at a pressure of $2 \times 10^{-5} \mathrm{mbar}$ for $20 \mathrm{~min}$; annealing the sample at $480^{\circ} \mathrm{C}_{\text {in }} \mathrm{CH}_{4}$ at a pressure of $2 \times 10^{-5} \mathrm{mbar}$ resulted in the formation of carbon clusters; further annealing the sample in the absence of $\mathrm{CH}_{4}$ at $550^{\circ} \mathrm{C}$ initiated the graphene nucleation; prolonged annealing without $\mathrm{CH}_{4}$ at higher temperature up to $720^{\circ} \mathrm{C}$ propelled the ripening of graphene islands.

Characterization of graphene in UHV LT-STM. The LT-STM experiments were carried out in a custom-built multichamber ultra-high-vacuum (UHV) system with base pressure better than $1.0 \times 10^{-10} \mathrm{mbar}$, housing an omicron LT-STM interfaced to a Nanonis controller. All STM imaging were performed at $77 \mathrm{~K}$ using constant current mode with an electrochemically etched tungsten tip. All the bias voltage was applied to the tip ${ }^{54}$

Structural models of clusters on $\mathrm{Cu}(110)$ surface. Stability of $\mathrm{C}_{1} \mathrm{H}_{\mathrm{x}}(0 \sim 4)$ or $\mathrm{C}_{2} \mathrm{H}_{\mathrm{x}}$ $(0 \sim 6)$ clusters on $\mathrm{Cu}(110)$ surface were studied using DFT calculations. A 5-layer slab with a $20 \AA$ vacuum layer was used as the substrate. The bottom layer was fixed to its bulk configuration and all other atoms were fully relaxed. A $(3 \times 4)$ supercell was chosen to make sure that clusters were separated to their neighboring clusters by more than $10 \AA$ A. In STM simulation, a $(2 \times 2)$ supercell was chosen according to the experimental coverage

Calculation details. All the calculations were performed using DFT implemented in the Vienna Ab Initio Simulation Package (VASP) within the generalized gradient approximation ${ }^{55,56}$ plus DFT-D2 van der Waals (vdW) correction ${ }^{57}$. The exchangecorrelation functional of Perdew-Burke-Ernzerhof ${ }^{58}$ and the projector-augmented wave ${ }^{59}$ methods were used. The plane-wave basis cutoff energy was set to $500 \mathrm{eV}$. The criteria of convergence for energy and force were set to $10^{-5} \mathrm{eV}$ and $0.02 \mathrm{eV} / \mathrm{A}$. For the $(3 \times 4)$ and $(2 \times 2)$ models, $(7 \times 7 \times 1)$ and $(10 \times 14 \times 1)$ k-point grids were used, respectively. STM images were simulated using the Tersoff and Hamann approximation ${ }^{47}$. The lattice parameter of bulk $\mathrm{Cu}$ was optimized to be $3.564 \AA^{60}$.

1. Novoselov, K. S. et al. Electric field effect in atomically thin carbon films. Science 306, 666-669 (2004).

2. Geim, A. K. \& Novoselov, K. S. The rise of graphene. Nat. Mater. 6, 183-191 (2007).

3. Geim, A. K. Graphene: Status and prospects. Science 324, 1530-1534 (2009).

4. Lee, C., Wei, X., Kysar, J. W. \& Hone, J. Measurement of the elastic properties and intrinsic strength of monolayer graphene. Science 321, 385-388 (2008).

5. Dan, Y., Lu, Y., Kybert, N. J., Luo, Z. \& Johnson, A. T. C. Intrinsic response of graphene vapor sensors. Nano Lett. 9, 1472-1475 (2009).

6. Stoller, M. D., Park, S., Zhu, Y., An, J. \& Ruoff, R. S. Graphene-based ultracapacitors. Nano Lett. 8, 3498-3502 (2008).

7. Stankovich, S. et al. Graphene-based composite materials. Nature 442, 282-286 (2006).

8. Sutter, P. Epitaxial graphene: How silicon leaves the scene. Nat. Mater. 8, 171-172 (2009).

9. Park, S. \& Ruoff, R. S. Chemical methods for the production of graphenes. Nat. Nanotechnol. 4, 217-224 (2009).

10. Stankovich, S. et al. Synthesis of graphene-based nanosheets via chemical reduction of exfoliated graphite oxide. Carbon 45, 1558-1565 (2007).

11. Li, D., Müller, M. B., Gilje, S., Kaner, R. B. \& Wallace, G. G. Processable aqueous dispersions of graphene nanosheets. Nat. Nanotechnol. 3, 101-105 (2008).

12. Wang, H. \& Hu, Y. H. Effect of oxygen content on structures of graphite oxides. Ind. Eng. Chem. Res. 50, 6132-6137 (2011)

13. Hernandez, Y. et al. High-yield production of graphene by liquid-phase exfoliation of graphite. Nat. Nanotechnol. 3, 563-568 (2008).

14. Loginova, E., Bartelt, N. C., Feibelman, P. J. \& McCarty, K. F. Factors influencing graphene growth on metal surfaces. New J. Phys. 11, 063046 (2009).

15. Wang, B., Ma, X., Caffio, M., Schaub, R. \& Li, W.-X. Size-selective carbon nanoclusters as precursors to the growth of epitaxial graphene. Nano Lett. 11, 424-430 (2011).

16. Lu, J., Yeo, P. S. E., Gan, C. K., Wu, P. \& Loh, K. P. Transforming $\mathrm{C}_{60}$ molecules into graphene quantum dots. Nat. Nanotechnol. 6, 247-252 (2011).

17. Coraux, J. et al. Growth of graphene on $\operatorname{Ir}(111)$. New J. Phys. 11, 023006 (2009).

18. Zhang, B. et al. Low-temperature chemical vapor deposition growth of graphene from toluene on electropolished copper foils. ACS Nano 6, 2471-2476 (2012).

19. Yu, Q. et al. Control and characterization of individual grains and grain boundaries in graphene grown by chemical vapour deposition. Nat. Mater. 10, 443-449 (2011).

20. Li, X. et al. Large-area graphene single crystals grown by low-pressure chemical vapor deposition of methane on copper. J. Am. Chem. Soc. 133, 2816-2819 (2011).

21. Bae, S. et al. Roll-to-roll production of 30-inch graphene films for transparent electrodes. Nat. Nanotechnol. 5, 574-578 (2010).

22. Bhaviripudi, S., Jia, X., Dresselhaus, M. S. \& Kong, J. Role of kinetic factors in chemical vapor deposition synthesis of uniform large area graphene using copper catalyst. Nano Lett. 10, 4128-4133 (2010).

23. Kim, K. S. et al. Large-scale pattern growth of graphene films for stretchable transparent electrodes. Nature 457, 706-710 (2009).

24. Gao, L. et al. Repeated growth and bubbling transfer of graphene with millimetresize single-crystal grains using platinum. Nat. Commun. 3, 699 (2012).
25. Huang, P. Y. et al. Grains and grain boundaries in single-layer graphene atomic patchwork quilts. Nature 469, 389-392 (2011)

26. Wood, J. D., Schmucker, S. W., Lyons, A. S., Pop, E. \& Lyding, J. W. Effects of polycrystalline $\mathrm{Cu}$ substrate on graphene growth by chemical vapor deposition. Nano Lett. 11, 4547-4554 (2011)

27. Lin, Y.-M. et al. 100-GHz Transistors from wafer-scale epitaxial graphene. Science 327, 662 (2010).

28. Rutter, G. M. et al. Scattering and interference in epitaxial graphene. Science 317, 219-222 (2007)

29. Marchini, S., Günther, S. \& Wintterlin, J. Scanning tunneling microscopy of graphene on Ru(0001). Phys. Rev. B 76, 075429 (2007).

30. Sutter, P. W., Flege, J.-I. \& Sutter, E. A. Epitaxial graphene on ruthenium. Nat. Mater. 7, 406-411 (2008)

31. Li, X., Cai, W., Colombo, L. \& Ruoff, R. S. Evolution of graphene growth on Ni and Cu by carbon isotope labeling. Nano Lett. 9, 4268-4272 (2009).

32. Vázquez de Parga, A. L. et al. Periodically rippled graphene: growth and spatially eesolved electronic structure. Phys. Rev. Lett. 100, 056807 (2008).

33. Luo, Z., Kim, S., Kawamoto, N., Rappe, A. M. \& Johnson, A. T. C. Growth mechanism of hexagonal-shape graphene flakes with zigzag edges. ACS Nano $\mathbf{5}$, 9154-9160 (2011).

34. Shu, H., Chen, X., Tao, X. \& Ding, F. Edge structural stability and kinetics of graphene chemical vapor deposition growth. ACS Nano 6, 3243-3250 (2012)

35. Gao, J., Yuan, Q., Hu, H., Zhao, J. \& Ding, F. Formation of carbon clusters in the initial stage of chemical vapor deposition graphene growth on $\mathrm{Ni}(111)$ surface. J. Phys. Chem. C 115, 17695-17703 (2011).

36. Mehdipour, H. \& Ostrikov, K. Kinetics of low-pressure, low-temperature graphene growth: toward single-layer, single-crystalline structure. ACS Nano 6 10276-10286 (2012).

37. Chen, H., Zhu, W. \& Zhang, Z. Contrasting behavior of carbon nucleation in the initial stages of graphene epitaxial growth on stepped metal surfaces. Phys. Rev. Lett. 104, 186101 (2010).

38. Zhang, W., Wu, P., Li, Z. \& Yang, J. First-principles thermodynamics of graphene growth on Cu surfaces. J. Phys. Chem. C 115, 17782-17787 (2011).

39. Riikonen, S., Krasheninnikov, A. V., Halonen, L. \& Nieminen, R. M. The Role of Stable and mobile carbon adspecies in copper-promoted graphene Growth. J. Phys. Chem. C 116, 5802-5809 (2012).

40. Gao, J., Yip, J., Zhao, J., Yakobson, B. I. \& Ding, F. Graphene nucleation on transition metal surface: structure transformation and role of the metal step edge. J. Am. Chem. Soc. 133, 5009-5015 (2011).

41. Zangwill, A. \& Vvedensky, D. D. Novel growth mechanism of epitaxial graphene on metals. Nano Lett. 11, 2092-2095 (2012).

42. Yuan, Q. et al. Magic carbon clusters in the chemical vapor deposition growth of graphene. J. Am. Chem. Soc. 134, 2970-2975 (2011).

43. Van Wesep, R. G., Chen, H., Zhu, W. \& Zhang, Z. Communication: Stable carbon nanoarches in the initial stages of epitaxial growth of graphene on $\mathrm{Cu}(111)$ J. Chem. Phys. 134, 171105-171104 (2011).

44. Niu, T., Zhou, M., Zhang, J., Feng, Y. \& Chen, W. Growth intermediates for CVD graphene on $\mathrm{Cu}(111)$ : carbon clusters and defective graphene. J. Am. Chem. Soc. 135, 8409-8414 (2013).

45. Mi, X., Meunier, V., Koratkar, N. \& Shi, Y. Facet-insensitive graphene growth on copper. Phys. Rev. B 85, 155436 (2012).

46. Lewis, B. \& Anderson, J. C. Nucleation and growth of thin films. Academic Press, London (1978).

47. Tersoff, J. \& Hamann, D. R. Theory of the scanning tunneling microscope. Phys. Rev. B 31, 805-813 (1985).

48. Wei, Y. et al. The nature of strength enhancement and weakening by pentagonheptagon defects in graphene. Nat. Mater. 11, 759-763 (2012).

49. Riedl, C., Coletti, C., Iwasaki, T., Zakharov, A. A. \& Starke, U. Quasi-free-standing epitaxial graphene on SiC obtained by hydrogen intercalation. Phys. Rev. Lett. 103, 246804 (2009).

50. Virojanadara, C., Watcharinyanon, S., Zakharov, A. A. \& Johansson, L. I. Epitaxial graphene on 6H-SiC and Li intercalation. Phys. Rev. B 82, 205402 (2010).

51. Oida, S. et al. Decoupling graphene from $\mathrm{SiC}(0001)$ via oxidation. Phys. Rev. B 82, 041411 (2010).

52. Wong, S. L. et al. Quasi-free-standing epitaxial graphene on $\mathrm{SiC}$ (0001) by fluorine intercalation from a molecular source. ACS Nano 5, 7662-7668 (2011).

53. Locatelli, A. et al. Temperature-driven reversible rippling and bonding of a graphene superlattice. ACS Nano 7, 6955-6963 (2013)

54. Chen, W. et al. Two-dimensional pentacene:3,4,9,10-perylenetetracarboxylic dianhydride supramolecular chiral networks on $\mathrm{Ag}(111)$. J. Am. Chem. Soc. 130 12285-12289 (2008).

55. Kresse, G. \& Furthmüller, J. Efficiency of ab-initio total energy calculations for metals and semiconductors using a plane-wave basis set. Comput. Mater. Sci. 6 $15-50$ (1996).

56. Kresse, G. \& Furthmüller, J. Efficient iterative schemes for ab initio total-energy calculations using a plane-wave basis set. Phys. Rev. B 54, 11169-11186 (1996).

57. Grimme, S. Semiempirical GGA-type density functional constructed with a longrange dispersion correction. J. Comput. Chem. 27, 1787-1799 (2006).

58. Perdew, J. P., Burke, K. \& Ernzerhof, M. Generalized gradient approximation made simple. Phys. Rev. Lett. 77, 3865-3868 (1996).

59. Kresse, G. \& Joubert, D. From ultrasoft pseudopotentials to the projector augmented-wave method. Phys. Rev. B 59, 1758-1775 (1999). 
60. Simon, N. J., Drexler, E. S. \& Reed, R. P. Properties of copper and copper alloys at cryogenic temperatures. International Copper Association, Ltd., New York and National Institute of Standards and Technology, Gaithersburg (1992).

\section{Acknowledgments}

Authors acknowledge the support from Singapore MOE grants R143-000-505-112, R143-000-530-112, R143-000-542-112, R143-000-559-112, NUS YIA grant R143-000-452-101, and NSFC funds 21173202 and 21222304.

\section{Author contributions}

W.C. conceived and designed the experiments; J.L.Z. and T.C.N. performed the experiments; Z.Z.W., S.N.W. and Z.Y.L. performed theoretical calculations; W.C. and J.L.Z. wrote the manuscript. All authors contributed to writing and revising the manuscript.

\section{Additional information}

Supplementary information accompanies this paper at http://www.nature.com/ scientificreports

Competing financial interests: The authors declare no competing financial interests. How to cite this article: Zhang, J.L. et al. Elementary Process for CVD Graphene on $\mathrm{Cu}(110)$ : Size-selective Carbon Clusters. Sci. Rep. 4, 4431; DOI:10.1038/srep04431 (2014).

(c) (7) This work is licensed under a Creative Commons Attribution 3.0 Unported license. To view a copy of this license, visit http://creativecommons.org/licenses/by/3.0 